\title{
Mortalidad por melanoma cutáneo en Colombia: estudio de tendencias
}

Mortality trends of cutaneous melanoma in Colombia

\section{María Alejandra García ${ }^{1}$}

1. Médica y cirujana de la Pontificia Universidad Javeriana, epidemióloga de la Universidad Autónoma de Madrid; residente de Otorrinolaringología, Fundación Universitaria de Ciencias de la Salud-Hospital de San José, Bogotá, D.C., Colombia.

\section{RESUMEN}

Introducción.El melanoma cutáneo representa un cáncer de gran letalidad entre los tumores de piel. Desde hace algunas décadas, el aumento rápido en el número de casos ha ocasionado que este cáncer se convierta en una prioridad para la salud pública a nivel mundial. A pesar de que la mayoría de la población Colombiana presenta un tipo de piel II-III según la escala de Fitzpatrick, Colombia es el cuarto país de América con mayor incidencia.

Materiales y métodos. Se utilizó la información de muertes por melanoma ocurridas entre el 2000 y el 2012 en Colombia, identificadas a través del registro de defunciones del Departamento Administrativo Nacional de Estadística (DANE). El total de muertes se estudió de manera global y por sexo. Se estimaron las tasas de mortalidad y su tendencia en el tiempo a nivel nacional, departamental y regional. Asimismo, se analizó el riesgo de muerte para cada año estudiado mediante el modelo de regresión de Poisson.

Resultados y conclusiones. Entre el 2000 y el 2012, 2.904 personas murieron por melanoma cutáneo en Colombia. El riesgo de muerte se elevó casi al doble durante el periodo, con una tasa ajustada por edad de 3,8 por millón de habitantes en el 2000 y de 6,6 en el 2012 (razón de tasas=1,75; IC ${ }_{95 \%}$ 1,4-2,1). El aumento anual de la mortalidad varió por región y sexo. En Colombia está aumentando la mortalidad por melanoma cutáneo. Para evitar su progresión es necesario crear estrategias integradas de educación como forma de prevención primaria y secundaria.

PALABRAS CLAVE: melanoma, epidemiología, tasa de mortalidad, Colombia, neoplasias cutáneas.

\section{ABSTRACT}

Background: Cutaneous melanoma is a highly lethal cancer within skin tumors. The rapid increase in total cases in the last decade has turned this cancer to be a priority for public health worldwide. Even though the majority of the Colombian population has a low-risk skin prototype, Colombia is the fourth country in America with higher incidence.

Materials and methods: The numbers of fatal cases of cutaneous mela- noma that occurred in Colombia from 2000 to 2012 was obtained from the mortality records of the Departamento Administrativo Nacional de Estadistica (DANE). Total mortalities were analyzed globally and according to age and according toage and
Correspondencia:

María Alejandra García

Email:

mgarciach11@gmail.com

Recibido: 16 de junio de 2016

Aceptado: 29 de agosto de 2016

No se reportan conflictos de interés. 
gender. Mortality rates and their tendency over time were estimated at a national, departmental and regional level; moreover, the relationship between mortality rates and date of death were analyzed using the Poisson regression model.

Results and conclusions: Between the years 2000 and 2012, 2,904 people died from cutaneous melanoma in Colombia. The mortality risk of cutaneous melanoma had a constant increase during this period; adjusted rate by age of 3.8 by the 2000 and 6.6 by 2012 (rate ratio=1.75; CI 95\% 1.4-2.1). The mortality-year rise varied depending on age, region and gender. In Colombia, mortality by cutaneous melanoma is increasing. In order to avoid its progression, it is necessary to create educational combined strategies as a tool for primary, as well as, secondary prevention.

KEY WORDS: Melanoma, epidemiology, mortality rate, Colombia, skin neoplasms.

\section{INTRODUCCIÓN}

El melanoma cutáneo es el tercer tumor de piel de mayor frecuencia después del carcinoma basocelular y el escamocelular. Sin embargo, es el más agresivo y letal, siendo el responsable de $80 \%$ de las muertes por estos tumores $(1,2)$.

La Organización Mundial de la Salud (OMS) a través de Globocan reportó 232.130 casos nuevos de melanoma con 55.488 muertes asociadas en el 2012 (3). Las tasas de incidencia y mortalidad más altas se encuentran en los países con predominio de raza caucásica. La historia de quemaduras solares y la exposición intermitente a la luz solar en la infancia son algunos de los principales factores de riesgo para esta enfermedad (4).

El aumento alarmante en el número de casos de melanoma cutáneo alrededor del mundo, único en su elevada mortalidad, ha ocasionado que este tumor haya pasado de ser una enfermedad poco frecuente a un problema de salud pública (5-7). Este fenómeno se ha relacionado con cambios en el estilo de vida y en los ideales de belleza de la población que incentivan prácticas de riesgo para el desarrollo de cáncer de piel (8).

En la mayoría de países el melanoma cutáneo es más frecuente en personas de piel blanca y sexo masculino (1). Sin embargo, se ha observado un aumento drástico de la incidencia en mujeres en los últimos años principalmente en la zona del tronco y las extremidades. Esto se ha asociado al uso rutinario de cámaras de bronceado, así como al de pequeñas prendas de vestir y mayor exposición solar (8). En algunos países se ha presenciado una elevación en la mortalidad en hombres acompañado con una estabilización en las tasas de mortalidad en mujeres (9). En parte esto podría deberse a que las mujeres realizan más prácticas de autocuidado de la piel (10).

Las personas mayores de 60 años presentan las tasas más altas de mortalidad por melanoma y su aumento persiste (1,11-14). En los países con mayor incidencia de melanoma, la diferencia entre incidencia y mortalidad tiende a ser amplia en los adultos jóvenes. Esto se debe a que la incidencia aumenta mientras que la mortalidad presenta estabilización o hasta un leve descenso (12,1520). Esta situación refleja la efectividad de las campañas de prevención que han permitido que cada vez se diagnostique en estadios más tempranos.

Colombia es el cuarto país de América con mayor incidencia de melanoma después de Estados unidos, Canadá y Uruguay. Sus tasas ajustadas alcanzan los 3,3 casos nuevos por 100.000 habitantes al año (3). A pesar de que la mayoría de la población colombiana presenta un prototipo de piel de bajo riesgo (21), en los últimos años se ha visto un aumento en las tasas de incidencia (11,22-23). El registro poblacional de cáncer de Cali, el más importante de Colombia, reportó tasas ajustadas de mortalidad que han venido en ascenso desde 1984 pues pasaron de ser para ese mismo año de 1,3 en hombres y 1,1 en mujeres, a 2,5 en hombres y 1,7 en mujeres para el 2013 (22).

La existencia de datos sobre melanoma en Colombia y en América Latina es escasa (24). El clima tropical de los países latinoamericanos favorecido por los cambios en el estilo de vida y la concepción de belleza, ha generado que esta población esté más expuesta a la radiación ultravioleta $(24,25)$.

Hasta el momento todos los estudios sobre mortalidad de melanoma en Colombia han sido regionales 
TABLA 1. Características de los pacientes fallecidos por melanoma cutáneo. Colombia, 2000-2012

\begin{tabular}{|l|l|l|}
\hline EDAD (AÑOS) & N & $\%$ \\
\hline $0-14$ & 12 & 0,41 \\
\hline $15-44$ & 407 & 14,02 \\
\hline $45-64$ & 967 & 33,30 \\
\hline $65-80$ & 967 & 33,30 \\
\hline 85 o más & 544 & 18,73 \\
\hline Sin información & 7 & 0,24 \\
\hline SEXO & 1.372 & 47,25 \\
\hline Mujeres & 1.532 & 52,75 \\
\hline Hombres & 2.904 & 100 \\
\hline TOTAL & & \\
\hline & & \\
\hline
\end{tabular}

o realizados en instituciones específicas $(26,27)$. Colombia es un país con múltiples etnias que difieren en el color de la piel y presenta una gran variabilidad geográfica, lo que dificulta la inferencia para el resto del país a partir de los resultados de regiones específicas. El objetivo de este estudio fue describir la evolución de la mortalidad por melanoma en el ámbito nacional y regional.

\section{MÉTODOS}

Se hizo un estudio descriptivo y retrospectivo. Los datos sobre mortalidad por melanoma cutáneo entre el 2000 y el 2012 se obtuvieron del Departamento Administrativo Nacional de Estadística (DANE). La información sobre la población en riesgo según año, edad, sexo y lugar de residencia, se extrajo de la proyección poblacional del DANE (28).

Se incluyeron todos los registros de mortalidad por melanoma cutáneo identificados con el código C43 (CIE-10) que fueran certificados por un médico. Se excluyeron los registros de los cuales no se tenía conocimiento del lugar de residencia. Para el cálculo de las tasas se incluyeron únicamente los registros de pacientes fallecidos con 20 años de edad o más.

Figura 1. Tasa Ajustada y Cruda de Mortalidad Por Melanoma Cutáneo. Colombia. 2000-2012.

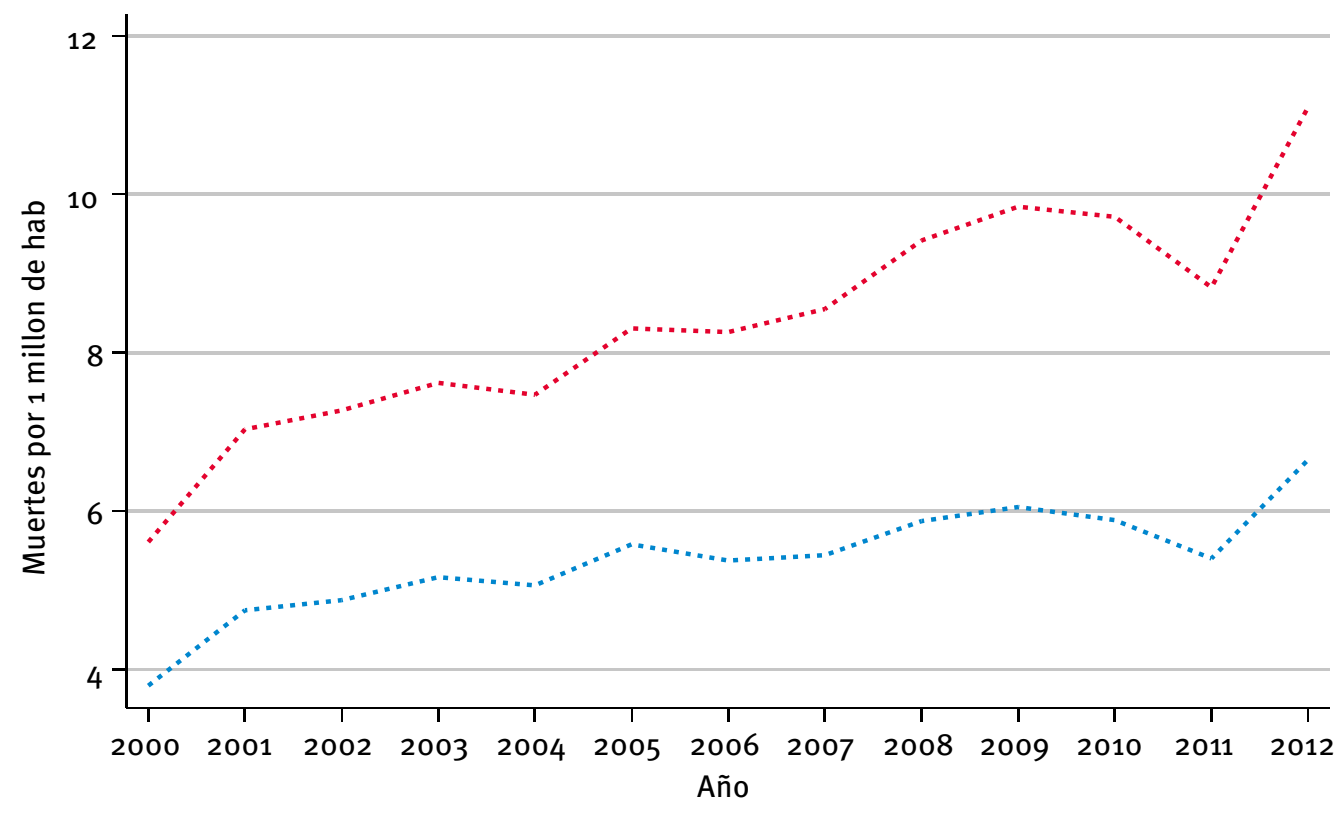


Se hizo el cálculo de la distribución de frecuencias de los datos para las variables de sexo, edad y departamento de residencia. Se calcularon las tasas crudas usando como numerador el número de fallecidos y como denominador la población en riesgo a mitad de periodo (28). Para el cálculo de las tasas ajustadas por edad se utilizó el método directo en base a los grupos etarios anteriormente descritos, usando como referencia la población mundial estándar de la OMS (29). Se excluyeron todos los fallecidos menores de 20 años debido a que el melanoma es muy raro en estos grupos de edad.

Para establecer las diferencias en las tasas de mortalidad por año, se calculó la razón de tasas comparando los diferentes años y utilizando el modelo de regresión de Poisson (30).

\section{RESULTADOS}

En Colombia hubo un total de 2.904 muertes por melanoma entre el 2000 y el 2012 (tabla 1) y las tasas crudas de mortalidad por melanoma pasaron de 5,6 a 11,1 muertes por un millón de habitantes en el mismo periodo (tabla 2, figura 1). Se evidenció un aumento sostenido de las tasas con el paso del tiempo, alcanzando para el año 2012 casi el doble de las cifras del 2000, con una razón de tasas=1,75 ( IC $_{95 \%}$ 1,4-2,1).

Los resultados muestran que las tasas en las mujeres son menores que en los hombres a lo largo de todo el periodo y que en ambos sexos las tasas crudas ascienden progresivamente, siendo hasta de 11 para hombres y de 8,5 para mujeres en el 2012 (figura 2).

Las tasas crudas y ajustadas de mortalidad en Colombia tienen una gran variabilidad. Bogotá, Risaralda, Caldas, Antioquia, Meta, Huila, Valle del Cauca, Quindío, Santander, Boyacá y Tolima son los departamentos con las tasas ajustadas más altas del periodo estudiado (tabla 3). Por otro lado, los departamentos de Atlántico, Bolívar, Chocó, La Guajira, San Andrés, Magdalena, Córdoba y Sucre no superan las 2 muertes al año por un millón de personas.

El análisis de datos por regiones muestra que más del $80 \%$ de las muertes provienen de la Región Andina (figura 3). Asimismo, los departamentos con tasas crudas de más de 8 muertes al año por un millón de personas se encuentran ubicados en la región andina y aquellos con tasas entre 4 y 8 muertes por millón de personas se encuentran en la región andina ó cerca de esta. Los departamentos con las tasas más bajas se encuentran en las zonas del Caribe, la Costa Pacífica, la Orinoquia y la Amazonia (tabla 4).

Figura 2. Tasa Cruda de Mortalidad por Melanoma Cutáneo según sexo. Colombia 2000-2012.

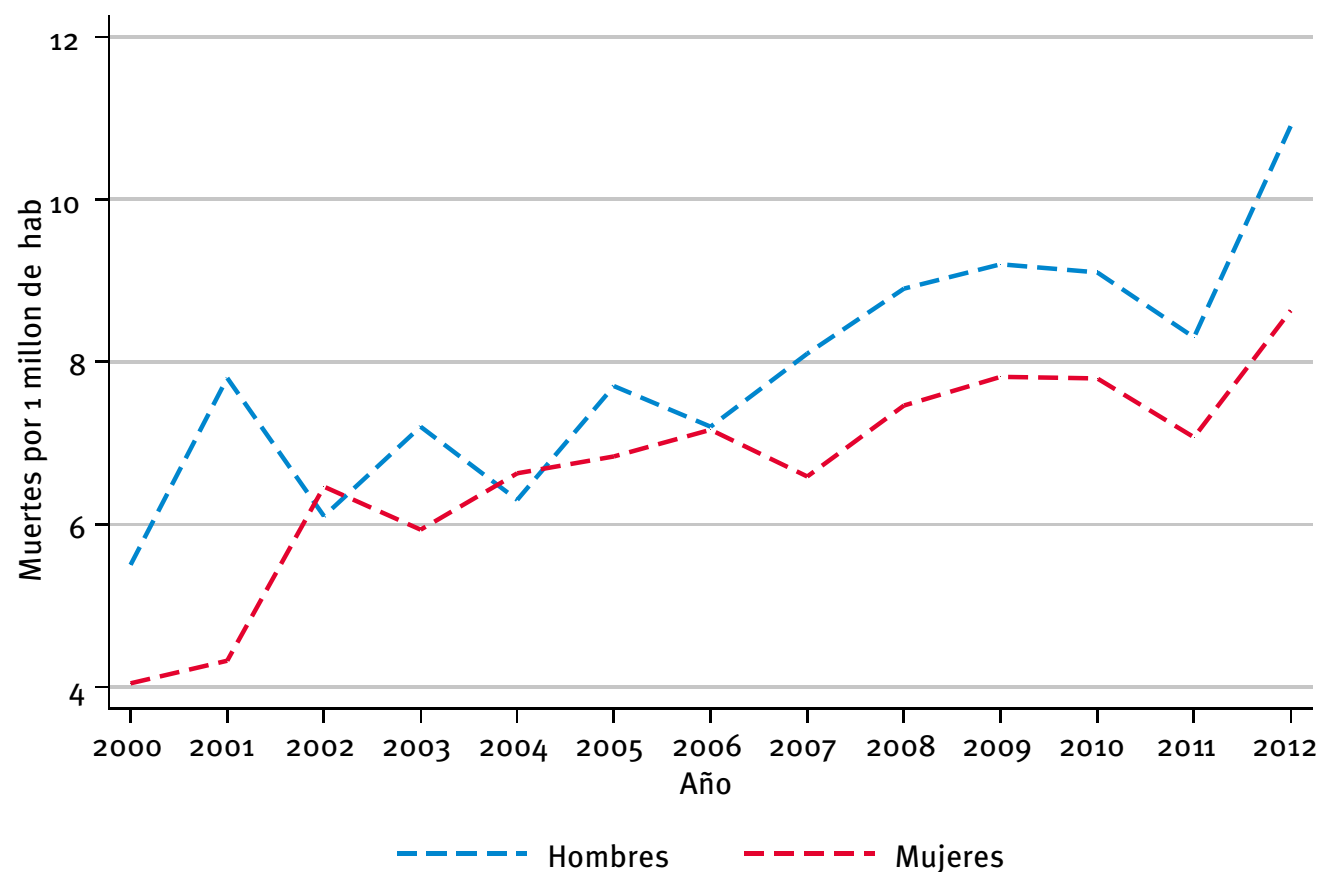


FIGURA 3. Porcentaje de Muertes por Melanoma Cutáneo según Región.

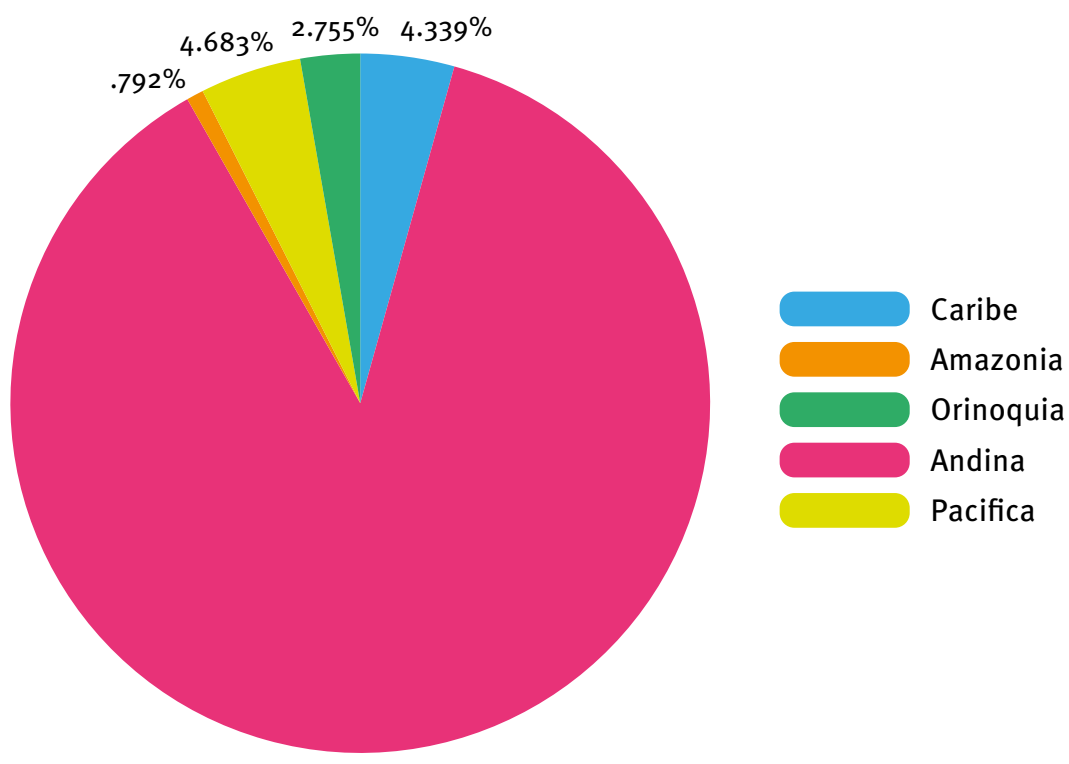

TABLA 2. Número de casos, tasas crudas y tasas ajustadas por edad de mortalidad por melanoma cutáneo en Colombia

\begin{tabular}{|c|c|c|c|c|c|}
\hline AÑO & CASOS & TASA AJUSTADA* + $^{*}$ & TASA CRUDA+ & $\mathbf{R T}^{*}$ & $\mathbf{P}$ \\
\hline 2000 & 129 & 3,8 & 5,6 & 1 & \\
\hline 2001 & 165 & 4,7 & 7,0 & $1,23(0,98-1,55)$ & 0,070 \\
\hline 2002 & 174 & 4,8 & 7,2 & $1,26(1,01-1,5)$ & 0,040 \\
\hline 2003 & 186 & 5,1 & 7,6 & $1,30(1,04-1,60)$ & 0,019 \\
\hline 2004 & 186 & 5,0 & 7,4 & $1,28(1,02-1,60)$ & 0,029 \\
\hline 2005 & 211 & 5,5 & 8,3 & $1,41(1,1-1,71)$ & 0,002 \\
\hline 2006 & 214 & 5,3 & 8,2 & $1,38(1,1-1,7)$ & 0,004 \\
\hline 2007 & 226 & 5,4 & 8,5 & $1,42(1,14-1,76)$ & 0,001 \\
\hline 2008 & 254 & 5,8 & 9,4 & $1,54(1,25-1,91)$ & 0,00 \\
\hline 2009 & 271 & 6 & 9,8 & $1,60(1,30-1,97)$ & 0,00 \\
\hline 2010 & 273 & 5,8 & 9,7 & $1,55(1,26-1,91)$ & 0,00 \\
\hline 2011 & 253 & 5,3 & 8,8 & $1,40(1,13-1,72)$ & 0,002 \\
\hline 2012 & 327 & 6,6 & 11,1 & $1,75(1,4-2,1)$ & 0,00 \\
\hline Total & 2869 & & & & \\
\hline \multicolumn{6}{|c|}{$\begin{array}{l}\text { ^ Tasa ajustada por grupos de edad } \\
\text { + Tasas en muertes por un millón de habitantes } \\
\text { RT: razón de tasas }\end{array}$} \\
\hline
\end{tabular}




\section{DISCUSIÓN}

En las últimas décadas la incidencia por melanoma cutáneo ha aumentado en muchos países alrededor del mundo, especialmente en los industrializados y donde predomina la raza caucásica. Esta situación se ha vuelto alarmante y ha transformado esta enfermedad en un problema de la salud pública que requiere especial atención (5).

El presente estudio es una aproximación a la situación de mortalidad por melanoma cutáneo en Colombia. Los resultados obtenidos son similares a aquellos previamente publicados con base en registros regionales o institucionales como el registro poblacional de Cali(26), el registro del Instituto Nacional de Cancerología y el del Centro Dermatológico Federico Lleras (31). En los cuales el aumento anual en el número de casos letales por melanoma cutáneo se hace evidente. Este fenómeno refleja un incremento en el número de casos diagnosticados con esta enfermedad que no han sido intervenidos tempranamente.

Existen algunas características que posiblemente se asocian con la tendencia desfavorable de la mortalidad por melanoma en Colombia. La primera es el cambio en los patrones de belleza, en los cuales la piel bronceada es sinónimo de bienestar lo que incentiva prácticas de exposición a la radiación solar (25). La segunda hace referencia a la falta de campañas educativas y preventivas que concienticen a la población sobre la problemática de esta enfermedad. Por último, al deterioro de la capa de ozono de la estratosfera por la emisión de gases que resulta en una exposición más intensa a los rayos ultravioleta, la cual es mayor en las ciudades de gran altitud (32).

Las ciudades ubicadas a mayor altura tienen mayor riesgo. Las tasas más altas de mortalidad por melanoma cutáneo se concentran en la región Andina, donde se encuentran ciudades con altitudes que varían entre 2.500 y 3.300 metros sobre el nivel del mar (33). Por otro lado, las regiones con las tasas más bajas se concentran en las zonas de la costa del Caribe, Pacífico y la selva amazónica, donde no se superan los 1.500 metros sobre el nivel del mar.

Las cifras muestran que en Colombia, al igual que en muchos países alrededor del mundo, la mortalidad por melanoma cutáneo es menor en las mujeres que en los hombres $(9,15,34)$. Esto ocurre posiblemente porque en las mujeres la mayoría de las lesiones tumorales se presentan en las extremidades donde son más fácilmente identificables. Además, las mujeres suelen consultar más tempranamente lo que favorece el diagnóstico de lesiones más delgadas con un mejor pronóstico (35).
TABLA 3. Número de casos y tasas ajustadas por edad de mortalidad por melanoma cutáneo según ente territorial, 2000-2012

\begin{tabular}{|c|c|c|c|}
\hline $\begin{array}{l}\text { ENTE } \\
\text { TERRITORIAL }\end{array}$ & CASOS & $\begin{array}{l}\text { TASA } \\
\text { CRUDA+ }\end{array}$ & $\begin{array}{l}\text { TASA } \\
\text { AJUSTADA* }^{*}\end{array}$ \\
\hline Antioquia & 517 & 9,4 & 6,9 \\
\hline Atlántico & 33 & 1,5 & 1,1 \\
\hline Arauca & 5 & 2,4 & 1,9 \\
\hline Bogotá, D.C. & 821 & 12,2 & 9,9 \\
\hline Bolívar & 21 & 1,24 & 0,9 \\
\hline Boyacá & 96 & 8,3 & 5,0 \\
\hline Caldas & 102 & 11,2 & 6,9 \\
\hline Caquetá & 12 & 3,3 & 3 \\
\hline Casanare & 16 & 6,02 & 6,4 \\
\hline Cauca & 50 & 4,2 & 3,2 \\
\hline Cesar & 15 & 1,8 & 1,6 \\
\hline Córdoba & 20 & 1,5 & 1,2 \\
\hline Cundinamarca & 137 & 6,3 & 4,4 \\
\hline Chocó & 6 & 1,6 & 1,4 \\
\hline Huila & 79 & 8,5 & 6,2 \\
\hline La Guajira & 5 & 0,8 & 0,7 \\
\hline Magdalena & 21 & 2 & 1,4 \\
\hline Meta & 59 & 8 & 6,7 \\
\hline Nariño & 80 & 5,6 & 4,1 \\
\hline $\begin{array}{l}\text { Norte de } \\
\text { Santander }\end{array}$ & 57 & 5 & 4,1 \\
\hline Putumayo & 5 & 1,9 & 1,9 \\
\hline Quindío & 45 & 8,8 & 5,7 \\
\hline Risaralda & 88 & 10 & 7,1 \\
\hline San Andrés & 0 & 0 & 0 \\
\hline Santander & 151 & 8 & 5,8 \\
\hline Sucre & 11 & 1,6 & 1,3 \\
\hline Tolima & 94 & 7,5 & 4,6 \\
\hline Valle del Cauca & 352 & 8,7 & 6,0 \\
\hline $\begin{array}{l}\text { Grupo de la } \\
\text { Amazonia }\end{array}$ & 6 & 2,4 & 2,6 \\
\hline \multicolumn{4}{|c|}{$\begin{array}{l}\text { ^ Tasa ajustada por edad } \\
+ \text { Muertes por un millón de personas-año }\end{array}$} \\
\hline
\end{tabular}


TABLA 4. Tasas crudas de mortalidad por ente territorial, Colombia, 2000-2012

\begin{tabular}{|c|c|}
\hline $\begin{array}{l}\text { TASA DE } \\
\text { MORTALIDAD* }\end{array}$ & ENTE TERRITORIAL \\
\hline$>8$ & $\begin{array}{l}\text { Antioquia, Bogotá, Risaralda, Caldas, Quindío, Valle del Cauca, Huila, } \\
\text { Boyacá }\end{array}$ \\
\hline$>4-8$ & $\begin{array}{l}\text { Meta, Santander, Tolima, Cundinamarca, Casanare, Nariño, Norte de } \\
\text { Santander, Cauca }\end{array}$ \\
\hline $0-4$ & $\begin{array}{l}\text { Caquetá, Arauca, Grupo de la Amazonia, Magdalena, Putumayo, Cesar, } \\
\text { Córdoba, Sucre, Chocó, Atlántico, Bolívar, La Guajira }\end{array}$ \\
\hline
\end{tabular}

Los resultados muestran un posible vínculo entre las variaciones de la mortalidad por melanoma cutáneo y el fototipo de piel de la población (21). Se sabe que las personas de piel oscura tienen un riesgo mucho menor de desarrollar melanoma que aquellas de piel blanca (36). En Colombia las regiones con menor mortalidad por melanoma cutáneo, como la costa Pacífica y Caribe, tienen una gran población de piel oscura. En la Amazonia la gran mayoría de la población son comunidades indígenas, con tipos de piel III-IV (37).

En cuanto a las limitaciones del estudio, es importante mencionar que los análisis están estrictamente basados en un registro de mortalidad en Colombia, el cual es la fuente de información de mortalidad más grande y homogénea que existe. Según los índices de calidad de la OMS, la cobertura de este registro alcanza el $80 \%$, lo que hace posible que estemos subestimando la mortalidad. Además, la mejoría de la cobertura a lo largo de los años pudo haber contribuido a un ascenso ficticio de las tasas de mortalidad.

\section{CONCLUSIONES}

La mortalidad por melanoma cutáneo ha aumentado en Colombia desde el año 2000 hasta el 2012 y la magnitud de este crecimiento varía según el sexo, la edad y el área geográfica. Para controlar esta situación y evitar su evolución, es necesario crear estrategias integradas de educación a la sociedad que generen una actitud más prudente frente a la exposición solar, así como de una mayor concientización sobre la importancia del autoexamen y el seguimiento frecuente por parte del especialista.

\section{REFERENCIAS}

1. Desmond RA, Soong S. Epidemiology of malignant melanoma. Surg Clin North Am. 2003;83:1-29.

2. Balch CM, Soong SJ, Gershenwald JE, Thompson JF, Reintgen DS, Cascinelli N, et al. Prognostic factors analysis of 17,600 melanoma patients: Validation of the American Joint Committee on Cancer melanoma staging system. J Clin Oncol. 2001;19:3622-34.

3. WHO. Globocan 2012, Melanoma of the skin-Estimated Incidence and Mortality. GLOBOCAN 2012: Estimate cancer Incidence, Mortality and Prevalence Worlwide in 2012. Fecha de consulta: 3 de julio de 2015. Disponible en: http://globocan. iarc.fr/Default.aspx

4. Gandini S, Sera F, Cattaruzza MS, Pasquini P, Picconi O, Boyle $\mathrm{P}$, et al. Meta-analysis of risk factors for cutaneous melanoma: II. Sun exposure. Eur J Cancer Oxf Engl 1990. 2005;41:45-60.

5. Garbe C, Leiter U. Melanoma epidemiology and trends. Clin Dermatol. 2009;27:3-9.

6. Glass AG, Hoover RN. The emerging epidemic of melanoma and squamous cell skin cancer. JAMA. 1989;262:2097-100.

7. Jemal A, Devesa SS, Hartge P, Tucker MA. Recent trends in cutaneous melanoma incidence among whites in the United States. J Natl Cancer Inst. 2001;93:678-83.

8. Héry C, Tryggvadóttir L, Sigurdsson T, Olafsdóttir E, Sigurgeirsson $\mathrm{B}$, Jonasson JG, et al. A melanoma epidemic in Iceland: Possible influence of sunbed use. Am J Epidemiol. 2010;172:762-7.

9. Jemal A, Devesa SS, Fears TR, Hartge P. Cancer surveillance series: Changing patterns of cutaneous malignant melanoma mortality rates among whites in the United States. J Natl Cancer Inst. 2000;92:811-8.

10. Miller DR, Geller AC, Wyatt SW, Halpern A, Howell JB, Cockerell C, et al. Melanoma awareness and self-examination practices: Results of a United States survey. J Am Acad Dermatol. 1996;34:962-70.

11. Tryggvadóttir L, Gislum M, Hakulinen T, Klint A, Engholm G, Storm HH, et al. Trends in the survival of patients diagnosed with malignant melanoma of the skin in the Nordic countries 1964-2003 followed up to the end of 2006. Acta Oncol Stockh Swed. 2010;49:665-72.

12. Hollestein LM, van den Akker SA, Nijsten T, Karim-Kos HE, Coebergh JW, de Vries E. Trends of cutaneous melanoma 
in The Netherlands: Increasing incidence rates among all Breslow thickness categories and rising mortality rates since 1989. Ann Oncol. 2012;23:524-30.

13. Jemal A, Saraiya M, Patel P, Cherala SS, Barnholtz-Sloan J, Kim J, et al. Recent trends in cutaneous melanoma incidence and death rates in the United States, 1992-2006. J Am Acad Dermatol. 2011;65:S17.e1-11.

14. Hoejberg L, Gad D, Gyldenkerne N, Bastholt L; Academy of Geriatric Cancer Research (AgeCare). Trends in melanoma in the elderly in Denmark, 1980-2012. Acta Oncol. 2016;55(Suppl.1):52-8.

15. Giblin A-V, Thomas JM. Incidence, mortality and survival in cutaneous melanoma. J Plast Reconstr Aesthetic Surg. 2007;60:32-40.

16. Baade $\mathrm{P}$, Coory M. Trends in melanoma mortality in Australia: 1950-2002 and their implications for melanoma control. Aust N Z J Public Health. 2005;29:383-6.

17. Downing A, Newton-Bishop JA, Forman D. Recent trends in cutaneous malignant melanoma in the Yorkshire region of England; incidence, mortality and survival in relation to stage of disease, 1993-2003. Br J Cancer. 2006;95:91-5.

18. Sneyd MJ, Cox B. A comparison of trends in melanoma mortality in New Zealand and Australia: The two countries with the highest melanoma incidence and mortality in the world. BMC Cancer. 2013;13:372.

19. Boniol M, Autier P, Gandini S. Melanoma mortality following skin cancer screening in Germany. BMJ Open. 2015;5:e008158.

20. Watson M, Geller AC, Tucker MA, Guy GP, Weinstock MA. Melanoma burden and recent trends among non-Hispanic whites aged 15-49years, United States. Prev Med. 2016;91:294-8.

21. Sánchez G, Nova J. Confiabilidad y reproducibilidad de la escala de fototipos de Fitzpatrick antes y después de un ejercicio de estandarización clínica. Biomédica. 2008;28:544-50.

22. Universidad del Valle. Registro Poblacional de Cáncer de Cali, Colombia. Melanoma, 1962-2010. RPCC: Registro Poblacional de Cáncer de Cali. Fecha de consulta: 30 de junio de 2015. Disponible en: http://rpcc.univalle.edu.co./es/index.php

23. Erdmann F, Lortet-Tieulent J, Schüz J, Zeeb H, Greinert R, Breitbart EW, et al. International trends in the incidence of malignant melanoma 1953-2008-are recent generations at higher or lower risk? Int J Cancer. 2013;132:385-400.

24. Schmerling RA, Loria D, Cinat G, Ramos WE, Cardona AF, Sánchez JL, et al. Cutaneous melanoma in Latin America: The need for more data. Rev Panam Salud Pública Pan Am J Public Health. 2011;30:431-8.

25. Chang C, Murzaku EC, Penn L, Abbasi NR, Davis PD, Berwick $\mathrm{M}$, et al. More skin, more sun, more tan, more melanoma. Am J Public Health. 2014;104:e92-9.

26. Oliveros C, Bravo JC, Zambrano A, Cepeda M, Ramírez AF. Tendencia de la incidencia y la mortalidad en melanoma maligno en los últimos 60 años y análisis de los datos del Registro Poblacional (sic.) de Cáncer de Cali. Rev Asoc Colomb Dermatol. 2012;20:255-64.

27. Pozzobon FC. Epidemiología del melanoma en el Instituto Nacional de Cancerología, 2006-2010. Bogotá, Colombia. Universidad Nacional de Colombia; 2012. Fecha de consulta: 5 de julio de 2015. Disponible en: http://www.bdigital.unal. edu.co/11513/1/05598712.2012.pdf

28. Departamento Administrativo Nacional de Estadistica (DANE). Proyeccion poblacional 1985-2020. DANE. Fecha de consulta: 3 de julio de 2015. Disponible en:http:// www.dane.gov.co/index.php/poblacion-y-demografia/ proyecciones-de-poblacion

29. Bray F. Cancer incidence in five continents. Age-standardization. Lyon: ARC Scientific Publications; 2002;8:87-9.

30. Hinde J. Compound poisson regression models. In: Gilchrist R, editor. GLIM 82: Proceedings of the International Conference on Generalised Linear Models.New York:Springer; 1982. p. 109-21.Fecha de consulta: 7 de julio de 2015. Disponible en:http://link.springer.com/cha pter/10.1007/978-1-4612-5771-4_11

31. Nova-Villanueva J, Sánchez-Vanegas G, Porras de Quintana L. Cáncer de piel: perfi 1 epidemiológico de un centro de referencia en Colombia 2003-2005. Revista de Salud Pública. 2007;9:595-601.

32. Aceituno-Madera P, Buendía-Eisman A, Olmo FJ, JiménezMoleón JJ, Serrano-Ortega S. Melanoma, altitud y radiación UVB. Actas Dermosifiliogr.2011;102:199-205.

33. Municipios de Colombia-Municipios.com.co [Internet]. Fecha de consulta: 15 de agosto de 2015. Disponible en:http:// www.municipios.com.co/

34. de Vries E, Bray FI, Coebergh JWW, Parkin DM. Changing epidemiology of malignant cutaneous melanoma in Europe 1953-1997: Rising trends in incidence and mortality but recent stabilizations in western Europe and decreases in Scandinavia. Int J Cancer. 2003;107:119-26.

35. Richardson B, Price A, Wagner M, Williams V, Lorigan P, Browne S, et al. Investigation of female survival benefit in metastatic melanoma. Br J Cancer. 1999;80:2025-33.

36. Crombie IK. Racial differences in melanoma incidence. Br J Cancer. 1979;40:185-93.

37. Departamento Administrativo Nacional de Estadistica (DANE). Colombia una nación multicultural-Su diversidad étnica. Bogotá: DANE; 2007. 